\title{
OPEN Surface model of the human red blood cell simulating changes in membrane curvature under strain
}

\begin{abstract}
Philip W. Kuchel ${ }^{1 \bowtie}$, Charles D. Cox ${ }^{2,3}{ }^{\text {, Daniel Daners }}{ }^{4}$, Dmitry Shishmarev ${ }^{5}$ \& Petrik Galvosas ${ }^{6}$
We present mathematical simulations of shapes of red blood cells (RBCs) and their cytoskeleton when they are subjected to linear strain. The cell surface is described by a previously reported quartic equation in three dimensional (3D) Cartesian space. Using recently available functions in Mathematica to triangularize the surfaces we computed four types of curvature of the membrane. We also mapped changes in mesh-triangle area and curvatures as the RBCs were distorted. The highly deformable red blood cell (erythrocyte; RBC) responds to mechanically imposed shape changes with enhanced glycolytic flux and cation transport. Such morphological changes are produced experimentally by suspending the cells in a gelatin gel, which is then elongated or compressed in a custom apparatus inside an NMR spectrometer. A key observation is the extent to which the maximum and minimum Principal Curvatures are localized symmetrically in patches at the poles or equators and distributed in rings around the main axis of the strained RBC. Changes on the nanometre to micro-meter scale of curvature, suggest activation of only a subset of the intrinsic mechanosensitive cation channels, Piezo1, during experiments carried out with controlled distortions, which persist for many hours. This finding is relevant to a proposal for non-uniform distribution of Piezo1 molecules around the RBC membrane. However, if the curvature that gates Piezo1 is at a very fine length scale, then membrane tension will determine local curvature; so, curvatures as computed here (in contrast to much finer surface irregularities) may not influence Piezo1 activity. Nevertheless, our analytical methods can be extended address these new mechanistic proposals. The geometrical reorganization of the simulated cytoskeleton informs ideas about the mechanism of concerted metabolic and cation-flux responses of the RBC to mechanically imposed shape changes.
\end{abstract}
Abbreviations
3D 3-Dimensional
NMR Nuclear magnetic resonance
RBC Red blood cell

Our aim is to convey a sense of scale in the distribution of proteins in the membrane and subjacent cytoskeleton, relative to the whole human red blood cell (RBC); and to graphically represent changes in membrane curvature on the $\sim 1 \mathrm{~nm}$ to $\sim 10 \mu \mathrm{m}$ scale, brought about by the systematic straining of these cells. This study was motivated by the quest for the geometrical and mechanistic basis of recent findings on mechanically distorted RBCs, made by using nuclear magnetic resonance (NMR) spectroscopy with stretched and compressed gels ${ }^{1,2}$.

The rates of glycolysis and transmembrane exchange of cations in RBCs are enhanced when these cells, suspended in gelatin gel, are stretched or compressed. The metabolic effect occurs only when the medium contains

\footnotetext{
${ }^{1}$ School of Life and Environmental Sciences, University of Sydney, Building G08, Sydney, NSW 2006, Australia. ${ }^{2}$ Victor Chang Cardiac Research Institute, Darlinghurst, Sydney, NSW, Australia. ${ }^{3}$ St Vincent's Clinical School, Faculty of Medicine, University of New South Wales, Sydney, NSW, Australia. ${ }^{4}$ School of Mathematics and Statistics, University of Sydney, Sydney, NSW, Australia. ${ }^{5}$ John Curtin School of Medical Research, Australian National University, Canberra, ACT, Australia. ${ }^{6} \mathrm{MacDiarmid}$ Institute for Advanced Materials and Nanotechnology, School of Chemical and Physical Sciences, Victoria University Wellington, Wellington, New Zealand. ${ }^{\square}$ email: philip.kuchel@sydney.edu.au
} 
$\mathrm{Ca}^{2+}$ ions; and the cells' responses are attributed to the activation of the mechanosensitive cation channel, Piezo1, when the plasma membrane is distorted ${ }^{3-7}$.

Cation-flux estimates via Piezol are typically made by electrophysiological means. This has been by classical patch clamping ${ }^{8}$, or in native RBCs via high throughput planar patch clamp measurements ${ }^{9}$. Because the electrophysiological measurements usually occur over seconds (of a transient response to shape change), while the NMR experiments can last for hours, a different property of channel opening and closure is in operation ${ }^{1,2,10}$. Under constant stretch, even for a channel that inactivates, a steady state current persists. With inactivating channels like Piezo1, this current is much smaller than the peak current generated by mechanical deformation. Thus, for long periods of stretch in NMR experiments, the results more closely correspond to the steady state Piezol current that occurs under constant mechanical load.

The Piezo1 three-arm (triskelion) structure spans a relatively large $\sim 23 \mathrm{~nm}$ diameter membrane patch; this has been measured from images obtained with cryo-electron microscopy ${ }^{11-13}$. Such imaging provides an indication of the likely values of curvature that are required to activate the channel. The current model suggests that the channel is curved in the resting state and flattens as membrane tension increases, which opens the channel ${ }^{14}$. Therefore, when inspecting the alteration of the average extent of membrane curvature, when an RBC is distorted, we might expect an increase in the fraction of the total membrane area that has lower curvature; since such altered topology (induced flatness) appears to cause activation of Piezol.

To quantify the alteration of membrane curvature that is brought about by stretching an idealized RBC, we employed a geometrical strain field, in which an RBC is elongated by a specified extent, denoted by $\xi$. In addition, we considered the variation of the angle of rotation of RBCs relative to the direction of the imposed strain field, prior to "switching the strain field on" (imposing stretch). This is relevant to describing the state-of-affairs of RBCs suspended in liquid gelatin prior to gelation, because the cells are known to have random orientations, which persist while in the gel state ${ }^{15}$. The mathematical model used in these analyses captures the key micro-anatomical dimensions of the discocyte that typify a human RBC (and in most other mammals, other than the camelidae) ${ }^{16}$.

Mathematical definitions of curvature of three dimensional (3D) surfaces are a major concern of differential geometry ${ }^{17,18}$; it is obvious that complicated formulae have become more accessible since the advent of symbolic computation, most notably in software packages like Mathematica ${ }^{19,20}$. Euler rotation matrices and strain tensors were applied to bring about the simulated RBC distortions (morphing), taking care to invoke the relevant inverse functions in the definition of the transformed shape function, and the curvature functions; again, Mathematica generated algebraic expressions symbolically. This remarkable outcome, despite the highly complicated forms, meant that the expressions were accurately evaluated to give estimates of curvatures. The ability to triangularize ${ }^{*}$ the mathematically specified surface of the RBC (in Mathematica) meant that the relative size of the cytoskeletal triangular (also referred to as hexagonal) mesh was able to be visualized in practicable computation times (minutes) ${ }^{19}$. ( ${ }^{*}$ Aside: we use the term triangularization as opposed to triangulation to distinguish the operation from the trigonometric procedure used in surveying, and cartography etc).

\section{Theory of methods}

RBC shape. There have been several expressions presented for the shape of the RBC including one based on the minimization of the bending energy of a dual layer membrane ${ }^{21}$. The mathematical expression for the $\mathrm{RBC}$ discocyte used here is close to those depicted $\mathrm{in}^{21}$, and it is a continuous degree- 4 surface that can be written either in Cartesian or disc-cyclide coordinates, making it versatile for numerical exploration ${ }^{16}$. The shape is constrained by three principal distances, the main diameter, $d$, the thickness at the centre of the dimples, $b$, and the maximum thickness (height) near the periphery of the cell, $h$ (see Fig. 1 for the first of many examples here):

$$
\left(x^{2}+y^{2}+z^{2}\right)^{2}+P\left(x^{2}+y^{2}\right)+Q z^{2}+R=0
$$

where,

$$
\begin{gathered}
P=-\frac{d^{2}}{2}+\frac{h^{2}}{2}\left(\frac{d^{2}}{b^{2}}-1\right)-\frac{h^{2}}{2}\left(\frac{d^{2}}{b^{2}}-1\right)\left(1-\frac{b^{2}}{h^{2}}\right)^{\frac{1}{2}} \\
Q=P \frac{d^{2}}{b^{2}}+\frac{b^{2}}{4}\left(\frac{d^{4}}{b^{4}}-1\right) \\
R=-P \frac{d^{2}}{4}-\frac{d^{4}}{16}
\end{gathered}
$$

The mean volume of a normal human RBC is $86 \mathrm{fL}$, while the surface area is variously stated to be $137 \pm 17$ or $143 \mu \mathrm{m}^{222,23}$. Thus, when $d=8 \mu \mathrm{m}, b=1 \mu \mathrm{m}$, and $h=2.12 \mu \mathrm{m}$, the model gives a volume of $86 \mathrm{fL}$ and a surface area of $128 \mu \mathrm{m}^{2}$. We gave precedence to the correct volume over the predicted smaller surface area in the above range of $120-154 \mu \mathrm{m}^{2}$.

Triangularization of the surface. While Eq. (1) is readily graphed in Mathematica, a representation of the cell's cytoskeleton requires partitioning the surface with a known number of struts (edges) in the geodeticdome-like closed polyhedron. In a human RBC, the struts of the mesh consist of head-to-head-associated two hetero-dimers of $\alpha$ - and $\beta$-spectrin, with junctional complexes of 12-14 actin monomers bound as short, twisted filaments. There are $\sim 121,000$ edges $^{24}$ so in Mathematica the Option, MaxCellMeasure in the BoundaryDiscre- 


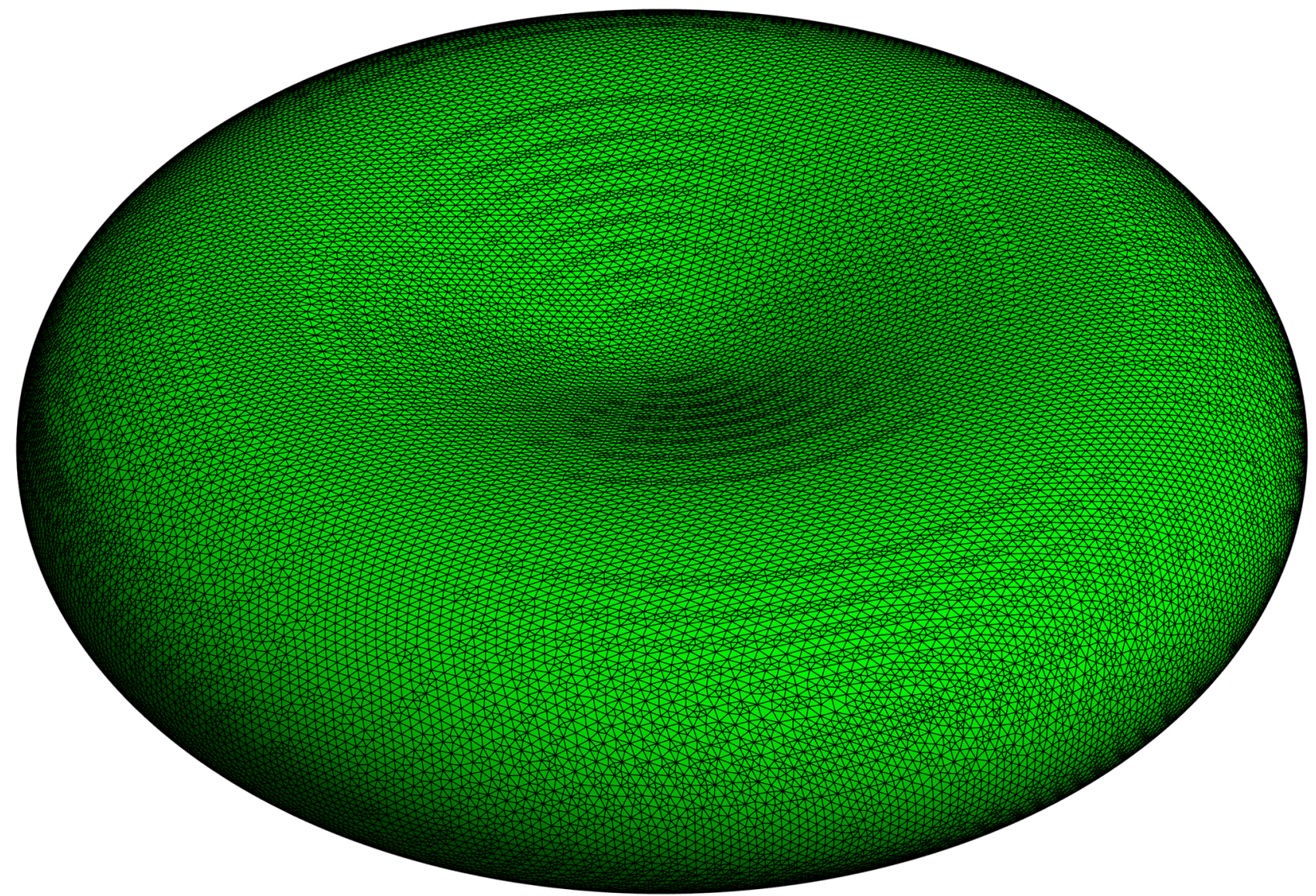

Figure 1. Triangular mesh of the RBC drawn to scale in its biconcave disc, using Eq. (1) in Mathematica. The main diameter $d=8 \mu \mathrm{m}$, thickness at the dimple $b=1 \mu \mathrm{m}$, and maximum height at the $\operatorname{rim} h=2.12 \mu \mathrm{m}$. The triangularization was with BoundaryDiscretizationRegion, applied to ImplicitRegion (Supplementary Information). Number of edges $=120,042$ and MeshCoordinates gave 40,136 points and 80,268 triangles. Green colouring was chosen over red (the natural choice for an RBC) for ease of visualizing the mesh. Notebook 2 has the Mathematica script used to generate this graphic.

tizeRegion function could be manually adjusted to make the number of edges in the polyhedron very close to this value (see the Supplementary Information for a Notebook implementation).

Shape transformation. We emulated the distortion of RBCs suspended in stretched gelatin gel by specifying that a geometrical strain field was applied in one direction, chosen to be along the $z$-axis. This equates to what applies in real NMR experiments ${ }^{1,2}$. It is not possible, experimentally, to align all the RBCs in gelatin media prior to or after gelation, so the members of the population of RBCs assume all possible orientations of their axes of symmetry in the strain field. Therefore, we considered three orientations as representative of all those that are possible (see "Discussion" for additional comments).

Euler rotation matrix. To alter RBC orientation, the independent variables in Eq. (1) were transformed, by rotation about the $x$-axis by an angle $\theta$, using an Euler rotation matrix ${ }^{25}$ :

$$
R_{\theta}=\left(\begin{array}{ccc}
1 & 0 & 0 \\
0 & \cos \theta & -\sin \theta \\
0 & \sin \theta & \cos \theta
\end{array}\right)
$$

where $\theta=0$ specifies the original orientation.

Strain tensor. This mathematical object is a matrix that invokes volume preserving elongation of a Cartesian body in the direction of the $z$-axis:

$$
S_{\xi}=\left(\begin{array}{ccc}
\frac{1}{\sqrt{\xi}} & 0 & 0 \\
0 & \frac{1}{\sqrt{\xi}} & 0 \\
0 & 0 & \xi
\end{array}\right)
$$


where $\xi=1$ specifies the original shape.

Inverse affine transformation of Eq. (1). The requisite overall coordinate-transformation matrix was derived symbolically in Mathematica with the following function (note the standard matrix product denoted by .).

$$
\operatorname{trf}=\text { InverseFunction }\left[\text { AffineTransform }\left[S_{\xi} \cdot R_{\theta}\right]\right] .
$$

followed by 'threading' the transformation through the discocyte expression (see the Supplementary Information for further explanation of the symbols and the complete Mathematica Notebook):

$$
\left(x^{2}+y^{2}+z^{2}\right)^{2}+p P\left(x^{2}+y^{2}\right)+q Q z^{2}+r R / . \operatorname{Thread}[\{x, y, z\} \rightarrow \operatorname{trf}[\{x, y, z\}]] / / \text { Simplify }
$$

and this yielded the shape-transformed Eq. (1):

$$
\begin{aligned}
& \frac{\left(z^{2}+\left(x^{2}+y^{2}\right) \xi^{3}\right)^{2}}{\xi^{4}} \\
& -\frac{\left(b^{6}+2 d^{4}\left(-1+\sqrt{1-\frac{b^{2}}{h^{2}}}\right) h^{2}+b^{2}\left(d^{4}-2 d^{2}\left(-1+\sqrt{1-\frac{b^{2}}{h^{2}}}\right) h^{2}\right)\right)\left(z \cos [\theta]-y \xi^{3 / 2} \sin [\theta]\right)^{2}}{4 b^{4} \xi^{2}} \\
& -\frac{\left(b^{2} d^{2}+\left(b^{2}-d^{2}\right) h^{2}-\left(b^{2}-d^{2}\right) \sqrt{1-\frac{b^{2}}{h^{2}}} h^{2}\right)\left(x^{2} \xi^{3}+\left(y \xi^{3 / 2} \cos [\theta]+z \sin [\theta]\right)^{2}\right)}{2 b^{2} \xi^{2}} \\
& -\frac{d^{4}}{16}+\frac{d^{2}\left(b^{2} d^{2}+\left(b^{2}-d^{2}\right) h^{2}-\left(b^{2}-d^{2}\right) \sqrt{1-\frac{b^{2}}{h^{2}}} h^{2}\right)}{8 b^{2}}=0
\end{aligned}
$$

Then, the triangularization of the surface was performed as follows:

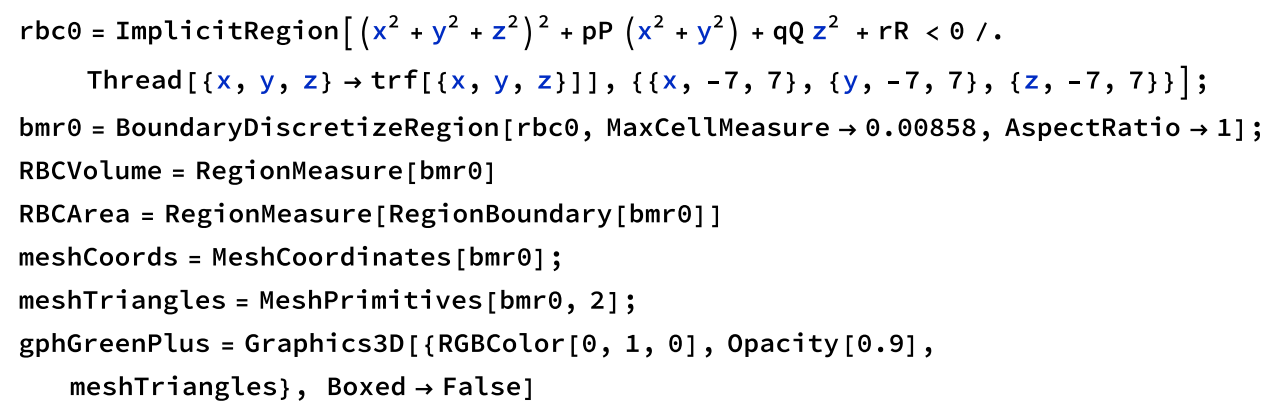

The function RegionMeasure applied to the boundary-discretized region gave the RBC volume; and the functions RegionMeasure[RegionBoundary[bmr0]] yielded the area of the RBC. The coordinates of the nodes of the mesh, and the list of all triangles, were obtained with the functions MeshCoordinates and MeshPrimitives. Finally, MeshTriangles was plotted by using Graphics3D (Fig. 1).

Curvature. Representing shape and curvature are primary objectives of differential geometry ${ }^{17}$, and modern computation with Mathematica provides a way of generating curvature expressions for surfaces that are defined implicitly by equations like Eq. (1). And, even more remarkably, those that are transformed to complicated expressions like Eq. (8).

A non-planar surface in three dimensions has a tangent plane and a normal vector at a specified point. In general, the curvature of the surface differs in one direction versus one at right angles to it. The shapes of these surfaces can be illustrated with the particular example of the hyperbolic paraboloid (saddle) ${ }^{17}$. Such a graphical rendering is shown in Supplementary Information, Fig. S1. The observation of, in general, two Principal Curvatures motivates the implementation of expressions that describe the curvature of the surface at a given point on the RBC. The fact that there are several ways of describing curvature of a surface may not be immediately obvious; but in general there are four expressions that have been explored in the theory of $3 \mathrm{D}$ differential geometry ${ }^{17}$.

Curvature expressions. We begin the presentation of the operations that are required to generate the types of curvatures of the RBC surface by defining $F[x, y, z]$ from Eq. (1):

$$
F[x, y, z] \equiv\left(x^{2}+y^{2}+z^{2}\right)^{2}+P\left(x^{2}+y^{2}\right)+Q z^{2}+R
$$

Four operations are to be carried out on $F[x, y, z]$ to make up the requisite expressions: (1) The gradient of $F, \nabla F[x, y, z]$, is a vector of partial derivatives of $F$ with respect to each of the independent Cartesian variables:

$$
\nabla F[x, y, z]=\left(F_{x}, F_{y}, F_{z}\right)
$$

(2) The Hessian of $F, H[F]$, is a $3 \times 3$ matrix of second order partial derivatives of $F$ : 


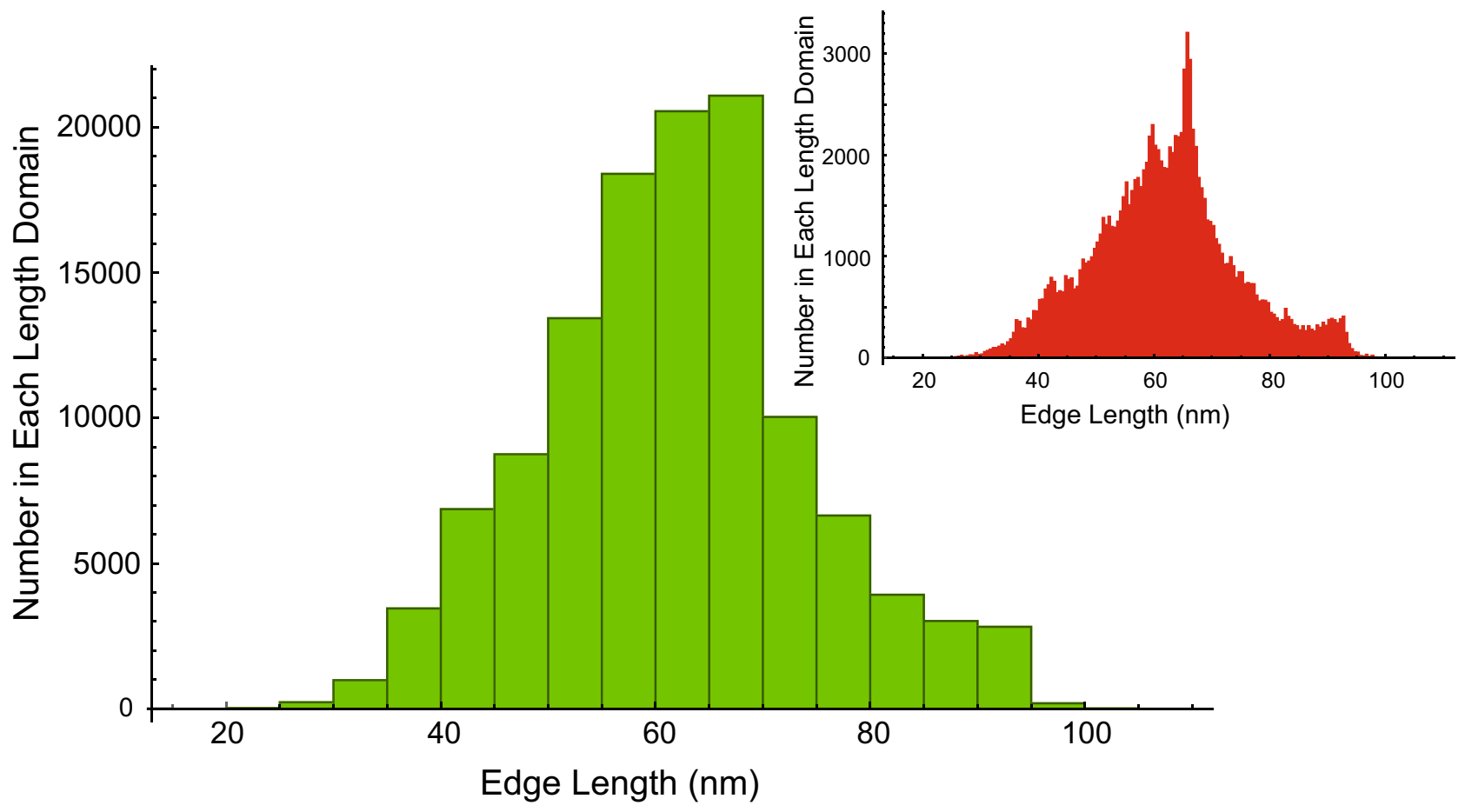

Figure 2. Data from the mesh in Fig. 1 showing the length-distribution histogram of edges in 20 bins (green); and, 200 bins (red inset). The mean edge length overall was $62 \mathrm{~nm}$. The median bin (tallest green pillar) contained $38 \%$ of the total bin contents and spanned $60-65 \mathrm{~nm}$; while the two most abundant bins spanning 60-70 nm contained 75\% of the edge lengths. Notebook 2 has the Mathematica script used to generate this graphic.

$$
H[F]=\left(\begin{array}{lll}
F_{x x} & F_{x y} & F_{x z} \\
F_{y x} & F_{y y} & F_{y z} \\
F_{z x} & F_{z y} & F_{z z}
\end{array}\right)
$$

(3) The cofactor or adjugate matrix, denoted by $H^{\star}[F]$ is defined as:

$$
H^{*}[F]=\left(\begin{array}{lll}
\operatorname{Cofactor}\left(F_{x x}\right) & \operatorname{Cofactor}\left(F_{x y}\right) & \operatorname{Cofactor}\left(F_{x z}\right) \\
\operatorname{Cofactor}\left(F_{y x}\right) & \operatorname{Cofactor}\left(F_{y y}\right) & \operatorname{Cofactor}\left(F_{y z}\right) \\
\operatorname{Cofactor}\left(F_{z x}\right) & \operatorname{Cofactor}\left(F_{z y}\right) & \operatorname{Cofactor}\left(F_{z z}\right)
\end{array}\right)
$$

where Cofactor $\left(F_{a b}\right), a, b=x, y$, or $z$, for each of the second derivatives in Eq. (13) is a matrix of determinants ${ }^{26}$.

(4) Finally, the trace of the Hessian matrix is required; this is simply the sum of the three terms in the leading diagonal of $H[F]$ [Eq. (13)]; it is the Laplace operator of $F$.

Gaussian curvature $K_{G}$. The Gaussian Curvature is expressed in terms of the vector of partial derivatives, its transpose, and the cofactor matrix of the Hessian ${ }^{17,18}$;

$$
\mathrm{K}_{\mathrm{G}}=\frac{\nabla F \cdot H^{*}[F] \cdot \nabla F^{\mathrm{T}}}{|\nabla F|^{4}}
$$

Mean curvature $K_{M}$. This is given by,

$$
\mathrm{K}_{\mathrm{M}}=\frac{\nabla F \cdot H[F] \cdot \nabla F^{\mathrm{T}}-|F|^{2} \operatorname{Trace}[H]}{2|\nabla F|^{3}}
$$

Principal curvatures $k_{1}$ and $k_{2}$. They are the largest and smallest curvatures at a given point. It appears obtuse to define the Principal Curvatures after the other two, but it is computationally more efficient to do so ${ }^{17,18}$ :

$$
\mathrm{k}_{1}=\mathrm{K}_{\mathrm{M}}+\sqrt{\mathrm{K}_{\mathrm{M}}^{2}-\mathrm{K}_{\mathrm{G}}}
$$




$$
\mathrm{k}_{2}=\mathrm{K}_{\mathrm{M}}-\sqrt{\mathrm{K}_{\mathrm{M}}^{2}-\mathrm{K}_{\mathrm{G}}}
$$

The relationships are $\mathrm{K}_{\mathrm{G}}=\mathrm{k}_{1} \mathrm{k}_{2}$, and $\mathrm{K}_{\mathrm{M}}=\frac{\mathrm{k}_{1}+\mathrm{k}_{2}}{2}$, the latter explaining the term Mean Curvature.

Implementation of Eqs. (15)-(18) for the RBC. Average at the three vertices. The next step after triangularization (e.g., Fig. 1) was to assign the values of curvature to each triangular face. This was done by applying Eqs. (14)-(17) to the vertices of each of the triangles and then averaging the three values.

Average at the centroid. An alternative treatment was to determine the positions of the centroid (centre of gravity) of each triangle and apply Eqs. (14)-(17) to those:

$$
\text { Centroid }[x, y, z]=(\operatorname{vertex} 1[x, y, z]+\operatorname{vertex} 2[x, y, z]+\operatorname{vertex} 3[x, y, z]) / 3
$$

Weighted average curvatures. Because the area of the triangles in any triangularization vary, as seen in the histogram of Fig. 2, the average of the curvatures of a set of triangles must be the weighted average. The weighting factor is the area of the triangle relative to the total area of all the triangles in the set:

$$
\text { wt AverageCurvature }=\frac{\sum_{i=1}^{N} \text { area }_{i} \text { curvature }_{i}}{\sum_{i=1}^{N} \text { area }_{i}}
$$

where the area of each triangle is given by the 'cross product formula' from vector analysis ${ }^{27}$. Specifically, the differences between the position vectors of each vertex, v1, v2, v3 are the side vectors of the triangle, $\times$ denotes the vector cross product, and || denotes the norm:

$$
\text { Area }=(1 / 2)|(\mathrm{v} 2-\mathrm{v} 1) \times(\mathrm{v} 3-\mathrm{v} 1)|
$$

\section{Results}

Triangularization. Euler test. The triangularization shown in Fig. 1 was closed (no gaps), with the number of edges E, faces (F; triangles) and vertices (V; mesh points) conforming to Euler's formula $\mathrm{F}-\mathrm{E}+\mathrm{V}=2^{28}$; the 2 is a topological invariant called the 'Euler characteristic' and is typically denoted by $\chi$.

Gauss-Bonnet theorem test. The Total Curvature, which is the integral of the values of the Gaussian Curvature, $\mathrm{K}_{\mathrm{G}}$, over a closed surface (like that used to describe the RBC), evaluates to $4 \pi$; specifically it is $2 \pi \chi^{17}$. This was indeed closely approximated by summing the product of $\mathrm{K}_{\mathrm{G}}$ (the mean of the three values of each triangle) and its area, across all triangles in the mesh. An example of the analysis is given in Notebook 5, Supplementary Information.

Triangles per mesh point. Detailed inspection of the mesh showed that in the vast majority of cases a single mesh point was met by six triangles; but there were a few instances of five and seven triangles sharing one vertex. These points appeared to be randomly dispersed on the surface. If the instances of such sharp triangles were high, this could cause problems with the finite element approximation of the surface area; but there was not a problem as noted above in relation to the Gauss-Bonnet Theorem test. On the other hand, such aberrant triangles are not a "good" representation of the spectrin mesh according to recent microscopic image analysis ${ }^{24,29}$.

Orientation. It was important to test the fidelity to the triangularization algorithm in generating the same RBC volume and area, and curvature estimates, when the RBC was rotated about the $x$-axis (and, by symmetry, any other rotations about lines through the origin in the $x, y$-plane) in the Cartesian coordinate system using Eq. (5). Specifically, the distribution of edge-lengths should not change when $\theta$ is varied, and this was reliably achieved.

Edge length. The next step was to compute the distribution of edge lengths, in order to study how these edges, which could be thought of as modelling the spectrin network, might imply that the spectrin is either stretched or compressed. Figure 2 shows the distribution of sides in Fig. 1, first at a resolution of 20 linearly spaced bins. The distribution is slightly skewed to the left, but it is unimodal; however, the inset which was based on 200 bins now appears to be at least trimodal (see "Discussion").

Triangle area distribution. Another feature of the surface triangularization, that is of biophysical importance, is the area of each triangle and whether this is consistent with the known span of membrane proteins that are corralled in the network. Figure S2a shows the separate triangularization of the RBC, which (as for Fig. 1) clearly indicates a range of shapes and sizes of the triangles. Sorting the triangles according to area showed a span from 0.0094 to $3431 \mathrm{~nm}^{2}$. When subdivided linearly into 10 bins it was seen that the 6 th bin contained the most triangles $(27,541)$ with a mean area of $1873 \mathrm{~nm}^{2}$; in other words $40.3 \%$ of the total area of $128 \mu \mathrm{m}^{2}$ had this mean area, while the mean area of a triangle across the whole cell was $1575 \mathrm{~nm}^{2}$. The size distribution is shown graphically in Fig. S3. 

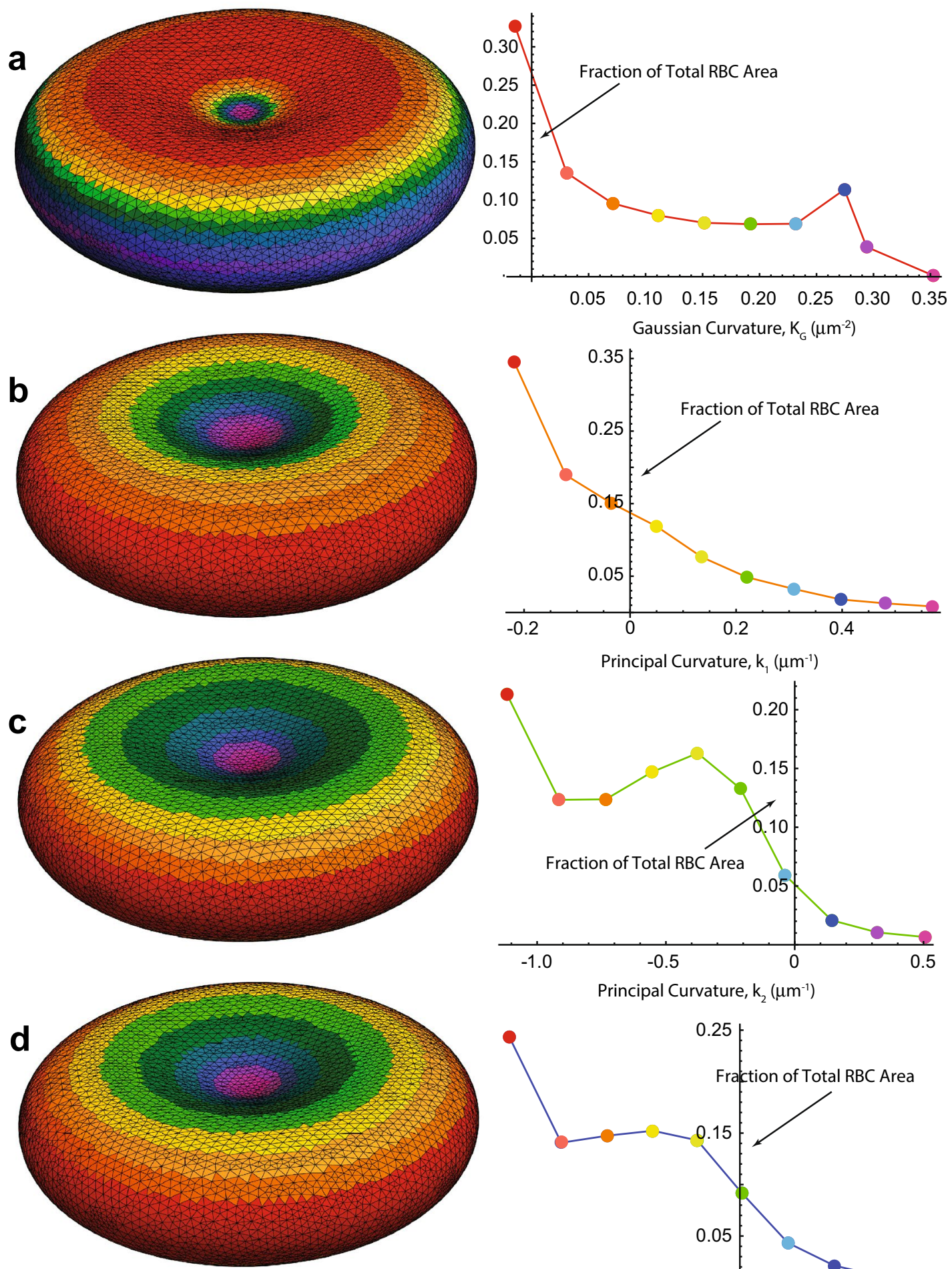

Range Key: Low to High
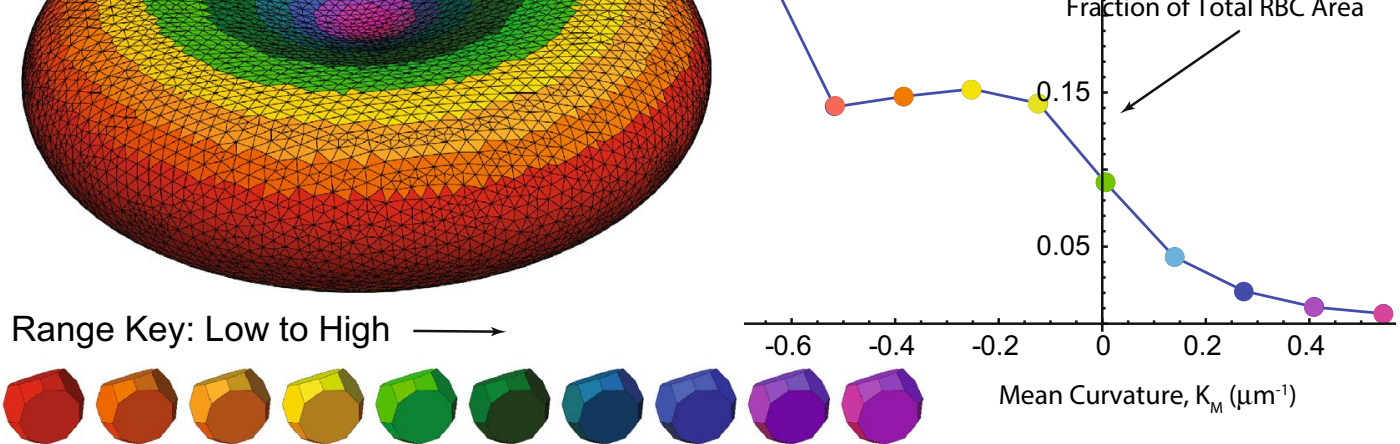

Mean Curvature, $\mathrm{K}_{\mathrm{M}}\left(\mu \mathrm{m}^{-1}\right)$

Figure 3. Positional dependence of the four types of curvature on the surface of the model RBC. (a) Gaussian Curvature, $\mathrm{K}_{\mathrm{G}}$; (b) Principal Curvature (maximum), $\mathrm{k}_{1}$; (c) Principal Curvature (minimum), $\mathrm{k}_{2}$; and (d) Mean Curvature, $K_{M}$. On the right of each cell is the graph of mean value (of the respective curvature) versus the fraction of the RBC area that has the curvature in a specified sub-domain of values. Specifically, the minimum and maximum values of each curvature were idenified, then the whole domain of values was divided linearly into 10 sub-domains (bins) with each assigned a colour-code, as shown in the given Range Key. The area of each triangle was computed ("Theory of Methods"; and Notebook 2), so the total area occupied by triangles in a given bin could be expressed as a fraction of the total $\mathrm{RBC}$ area, $128 \mu \mathrm{m}^{2}$. For speed of computation the triangularization was made with fewer mesh points than for Fig. 1, specifically 13,640 triangles and 6822 mesh points. 
Curvature mapping. A primary aim was to devise a means of displaying (mapping) the distribution of curvature(s) on the surface of the RBC. For this, a colour-coding program was written. Each triangle from the triangularization was stored in one of 10 value domains, according to whichever of the attributes was to be mapped. Figure 3 shows an undistorted RBC with its axis of symmetry normal to the the $x, y$-plane, and for which the average of the curvatures at each of the three vertices of each triangle was assigned.

Rotation and strain. The affine transformation, which combines both rotation and strain, produced shape changes such as those shown in Fig. 4 . For this figure the RBC was rotated by $45^{\circ}$ from the $x$, $y$-plane and then increasingly stretched.

On stretching (in the z-direction) the RBC was elongated and became narrower, an effect best seen in the overlapping images in Fig. 4e. However, the width of the RBC at the dimples and the maximum curvature at the rim both increased, as if the opposite faces of the cell were being pulled apart.

Volume and surface area during distortion. The surface area of an RBC declines with age in the blood circulation $^{30,31}$.

It is also known that an RBC's surface area cannot be increased by more than $\sim 15 \%$ before it ruptures; this was discovered in studies with RBCs swelling in hypotonic media ${ }^{32}$. In our own experiments with RBCs suspended in gelatin gel that is then stretched, haemolysis is very extensive with two-fold stretching $(\xi=2)$ (unpublished results). Therefore, it was important to explore the volume and surface area inter-relationships that are brought about by the affine transformation [Eqs. (5) and (6)].

Figure 5a shows that the surface area decreases on stretching if the RBC lies across the strain field; but it increases by up to $21 \%$ as the cell is stretched by $75 \%(\xi=1.75)$ when aligned with its disc-plane parallel to the strain field. Meanwhile, the volume of the RBC scarcely changed under all the angles of orientation and extents of stretching explored herein (Fig. 5b).

Overall, we concluded from Fig. 5 that RBCs sustain increased or decreased surface area depending on their initial orientation in the gel on stretching the sample, while the volume did not change significantly. The extent of area change is much less than would occur with a sphere; and this helps explain why the biconcave disc shape has been naturally selected. Specifically, the particular shape enables volume and surface area preservation during passage of the RBC through the capillaries of the peripheral tissues and lungs s $^{2,30,31,33}$.

Colour-coded curvatures for different values of $\boldsymbol{\theta}$ and $\boldsymbol{\xi}$. Figure 6 shows RBCs that were tilted at $45^{\circ}$ around the $x$-axis and progressively strained from no extension to a maximum of $\xi=1.75$. The changes in curvature are indicated by the changes in colour; the most notable feature for the Gaussian curvature $\left(\mathrm{K}_{\mathrm{G}}\right)$ is the increasing dominance of areas of red denoting increased area of lower values as the RBCs are stretched. On the other hand, the intermediate values (green) dominate the area of the values of $\mathrm{k}_{1}$ (the maximum Principal Curvature). Numerous other comparisons can be made, as are taken up in the "Discussion".

Another way of depicting the changes in curvature with orientation and strain is via a form of histogram shown in Fig. 7. The graphs show the fraction of the RBC area that is occupied by triangles with curvature (for each of the four types) in the neighbourhood of the mean values that correspond to 10 bins, arranged uniformly between the minimum and maximum values of the respective curvature.

The values of the curvatures span different ranges in all scenarios of orientation and strain. When there was no strain (top row of Fig. 7, and Fig. 3) the triangles with $\mathrm{k}_{1}$ (orange line) of smallest value occupied the largest area. When the RBCs at $0^{\circ}$ were stretched the minimum of $\mathrm{k}_{1}$ decreased in value but occupied a much larger area of the cell as stretching was increased. Another notable feature at $45^{\circ}$ orientation was the shift in $\mathrm{k}_{2}$ and the Mean Curvature (green and blue lines) to larger values as stretching was increased, with the maximum area occupied by triangles of intermediate values. Also, there is a clearly defined maximum value in these polygonal graphs. For the RBCs at $90^{\circ}$ orientation $k_{1}$ and $k_{2}$ both decreasd with increased stretching, with the maximum area occupied by large values. Overall, the patterns of all four polygonal graphs shifted progressively as stretching was increased, but at each of the angles of orientation the patterns were distinctly different.

This is but a snapshot of RBCs orientated at three angles; for a continuous distribution of angles, we would expect a smooth transition from the left hand column of polygonal graphs through the middle column to the right hand column.

Edge length distribution as a function of extent of stretching. Figure 8 shows that for $\theta=0^{\circ}$ the median value of edge length of the triangularization decreased on stretching by $75 \%$ more than the original value. This is consistent with the fact that Fig. 5 shows that for $\theta=0^{\circ}$ the surface area decreased with increasing $\xi$. The main feature for the RBCs at $45^{\circ}$ is the emergence of a broad bimodal distribution of edge lengths that is most clearly evident in the bottom of the middle column of the histograms. On the other hand, when the RBCs were at $90^{\circ}$, the distribution of edge lengths remained relatively narrow for stretching all the way to $\xi=1.75$. From Fig. 5, it is at this orientation that most increase in area took place, and it is especially clear in the bottom right-hand histogram that the median edge length was $\sim 70 \mathrm{~nm}$; this is similar to the second maximum in the second column. Consistent with this observation is that both RBC orientations displayed increases in surface area as well (Fig. 5). 
a

b

C

d

e

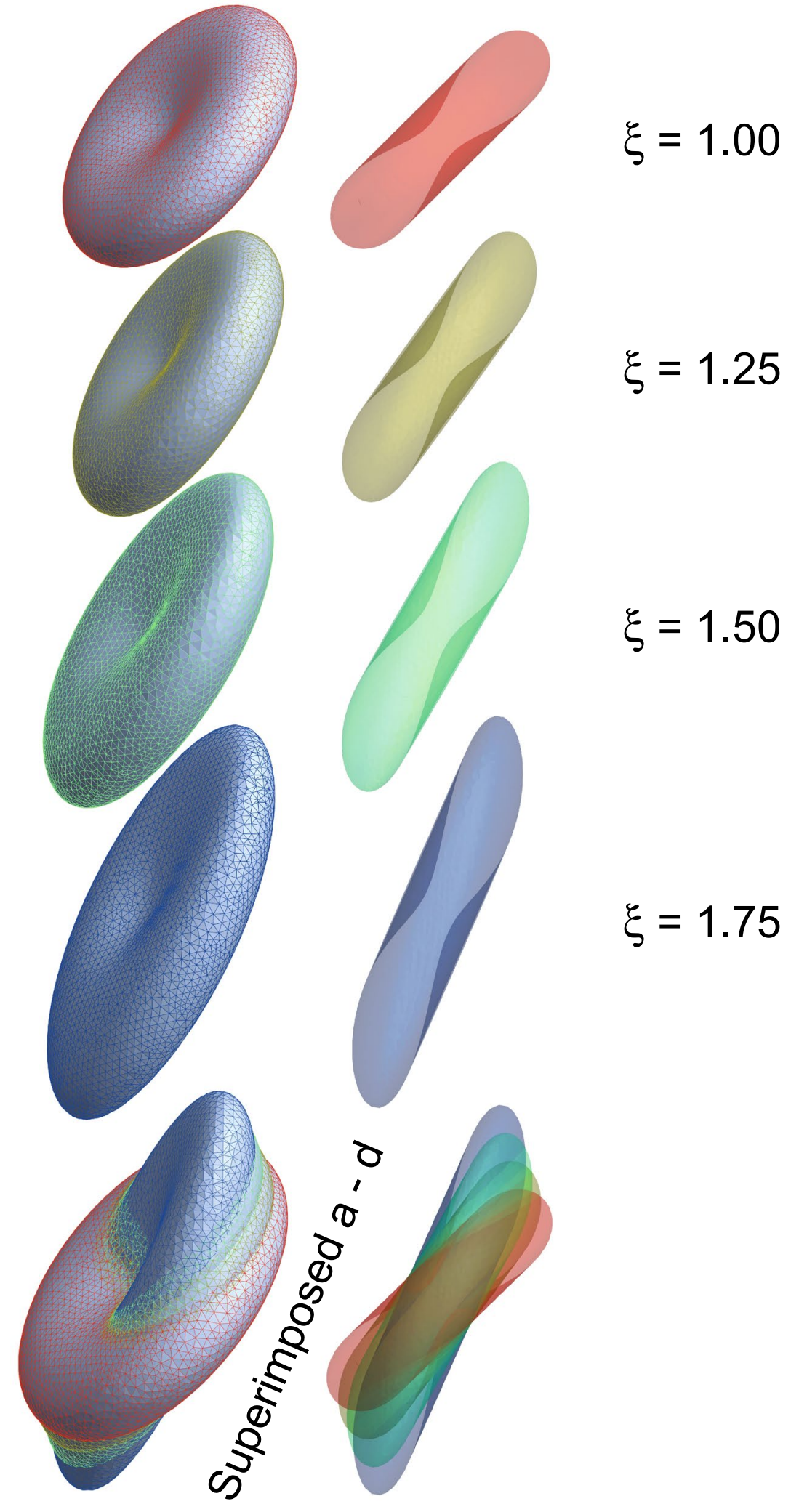

Figure 4. RBC rotated through $45^{\circ}$ about the $\mathrm{x}$-axis, relative to a linear strain field imposed in the $z$-direction. The relaxed RBC (a) had the same dimensions as in Fig. 1 (in fact, as in all the figures in this article): (a) No elongation, $\xi=1.00$; (b) stretched by $25 \%, \xi=1.25$; (c) stretched by $50 \%, \xi=1.50$; (d) stretched by $75 \%, \xi=1.75$; and (e) showing the relative elongation and concomitant narrowing of the RBCs by superimposing the images. Colour coding was used to provide distinction between the RBCs in (e). In the boundary discretization MaxCellMeasure was set to 0.1 giving $~ 8000$ mesh points. 


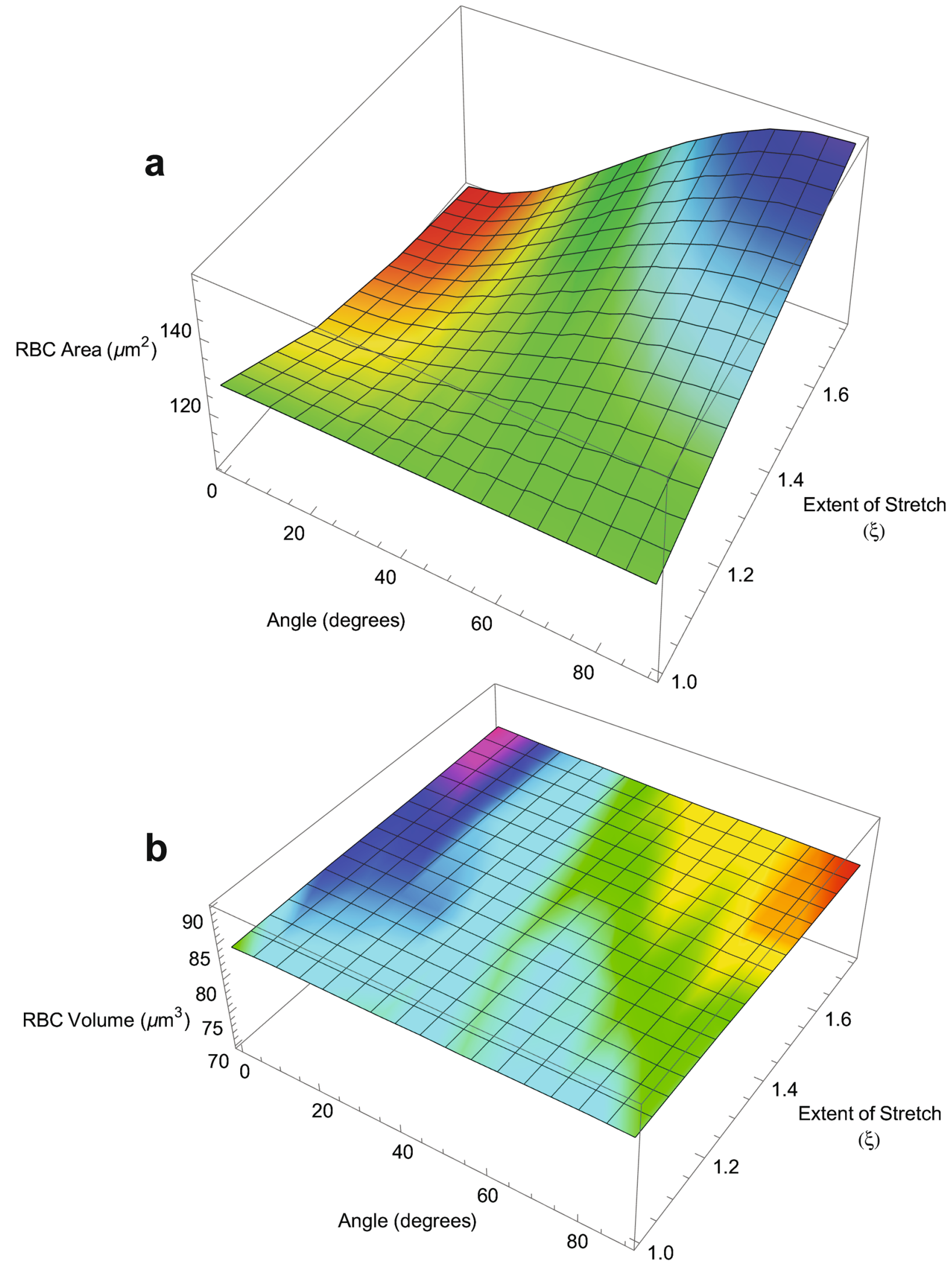

Figure 5. Dependence of RBC surface area (a) and volume (b) on rotation about to the $x$-axis $(\theta)$ and stretching along the $z$-axis $(\xi)$. The relaxed RBC had the same dimensions as in Fig. 1. 


\section{Extent of Stretching in z-Direction $(\xi)$}
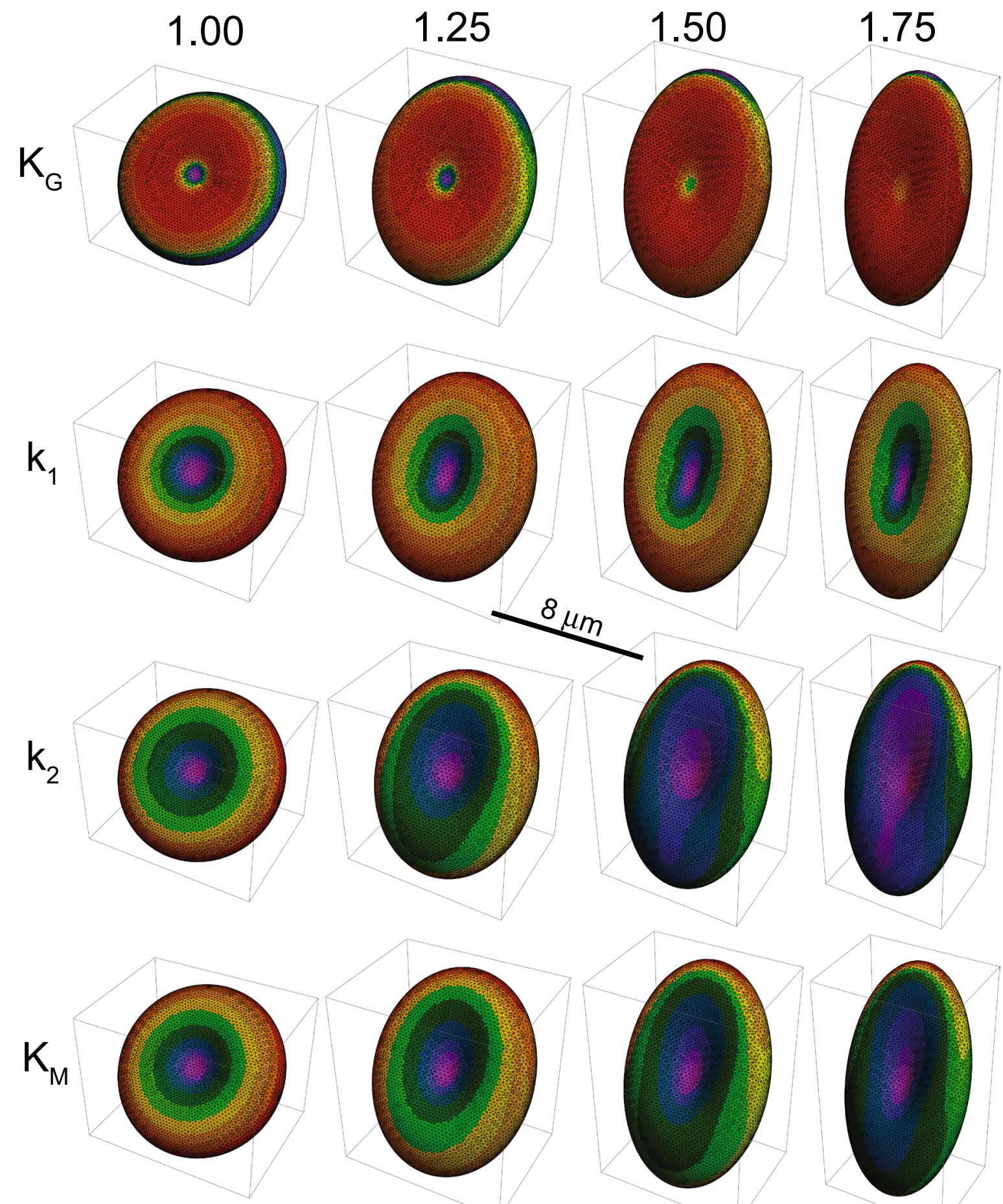

Range Key: Low to High
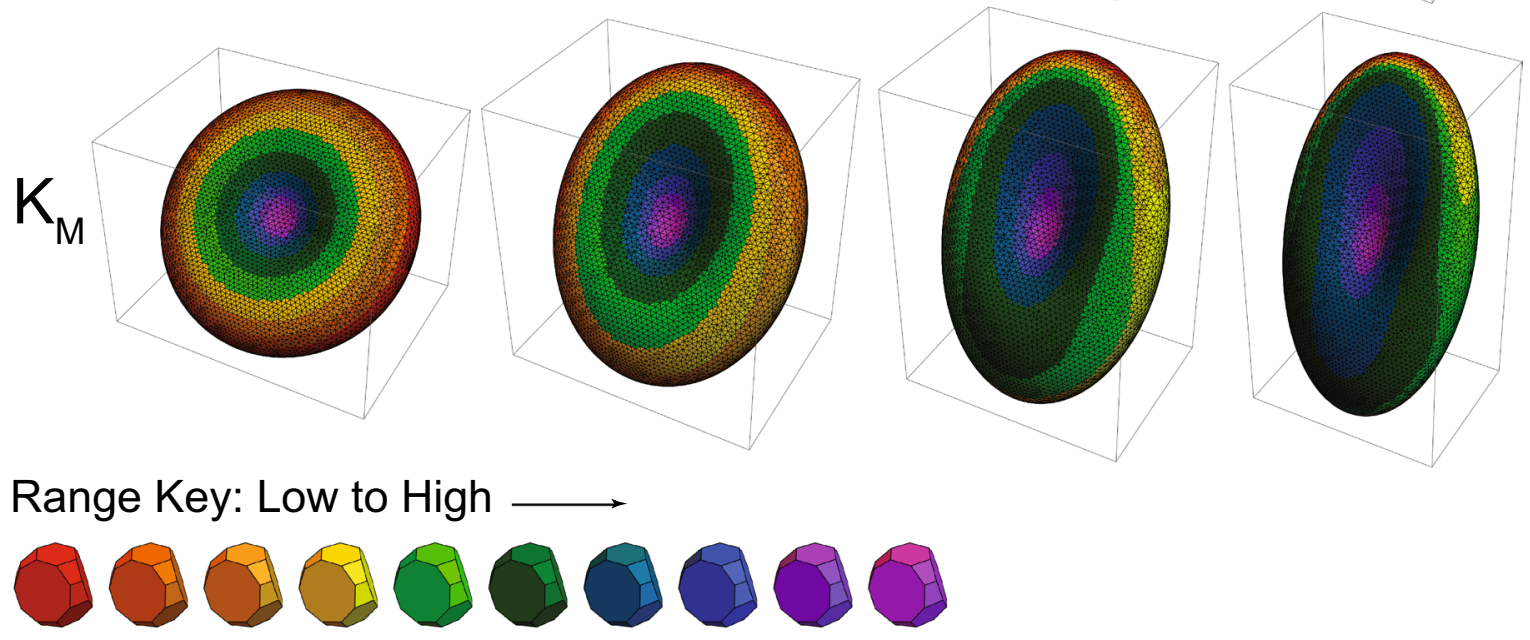

Figure 6. Curvatures of $\mathrm{RBC}$ rotated $45^{\circ}$ around the $x$-axis and stretched in the $z$-direction by the extents $(\xi)$ indicated above. The relaxed RBC had the same dimensions as in Fig. 1. The central scale bar indicates $d=8 \mu \mathrm{m}$, the main diameter of the fully relaxed RBC. $\mathrm{K}_{\mathrm{G}}$ denotes Gaussian Curvature; $\mathrm{k}_{1}$, Principal Curvature (maximum); $\mathrm{k}_{2}$, Principal Curvature (minimum); and $\mathrm{K}_{\mathrm{M}}$, Mean Curvature. The values of the respective curvatures across the 10 subdivisions of the domain of values are those shown in the central column of the polygonal graphs in Fig. 7. 


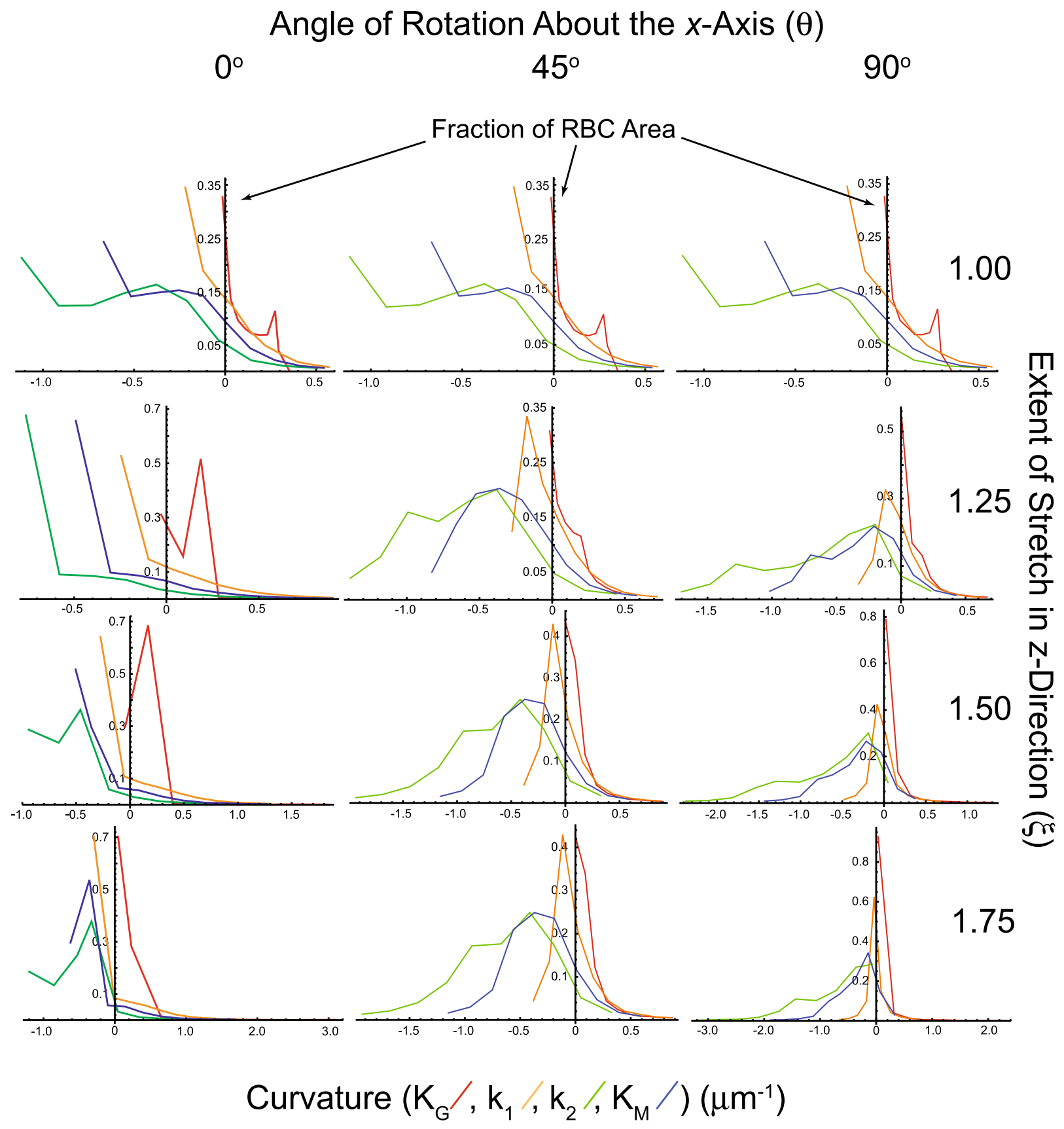

Figure 7. Curvature graphs of an $\mathrm{RBC}$ rotated at $0^{\circ}, 45^{\circ}$ and $90^{\circ}$ around the $x$-axis and stretched in the $z$-direction by the extents $(\xi)$ indicated on the right. The colours of the polygonal plots indicate: Gaussian Curvature $\mathrm{K}_{\mathrm{G}}\left(\mu \mathrm{m}^{-2}\right)$, red; Principal Curvature (maximum), $\mathrm{k}_{1}$, orange; Principal Curvature (minimum), $\mathrm{k}_{2}$, green; and Mean Curvature $\mathrm{K}_{\mathrm{M}}$, blue. Each discontinuity of the polygons denotes the mean value in curvature of the bin in the given curvature domain.

\section{Discussion}

Triangularization. The fully extended $\alpha$ - and $\beta$-spectrin heterotetramer has an end-to-end length of $\sim 200 \mathrm{~nm}^{24}$; but the filaments of unstretched RBC membranes have an average distance, between the nodal F-actin helices, of $60-70 \mathrm{~nm}^{34}$ and up to $85 \mathrm{~nm}^{29}$. The automatic triangularization that we used here to model the RBC cytoskeleton was controlled to correspond to $121,000 \alpha$-and $\beta$-spectrin heterotetramers; and this gave $\sim 65 \mathrm{~nm}$ as the mean value of the distance between two neighbouring nodes. This would imply that native spectrin has its filaments in a bent or serpentine form that more than halves the end-to-end distance of the tetramer. This state of the struts could be maintained by the central complex coordinated by ankyrin- $1^{24}$ imposing curvature on them.

Given that the fully extended struts of the cytoskeleton are $\sim 200 \mathrm{~nm}$ long ${ }^{24}$, basic geometry (triangle side $a$ gives area $=a^{2} \sqrt{3} / 4$ ) yields an estimate of the area of the resulting tessellation as follows: a single equilateral 


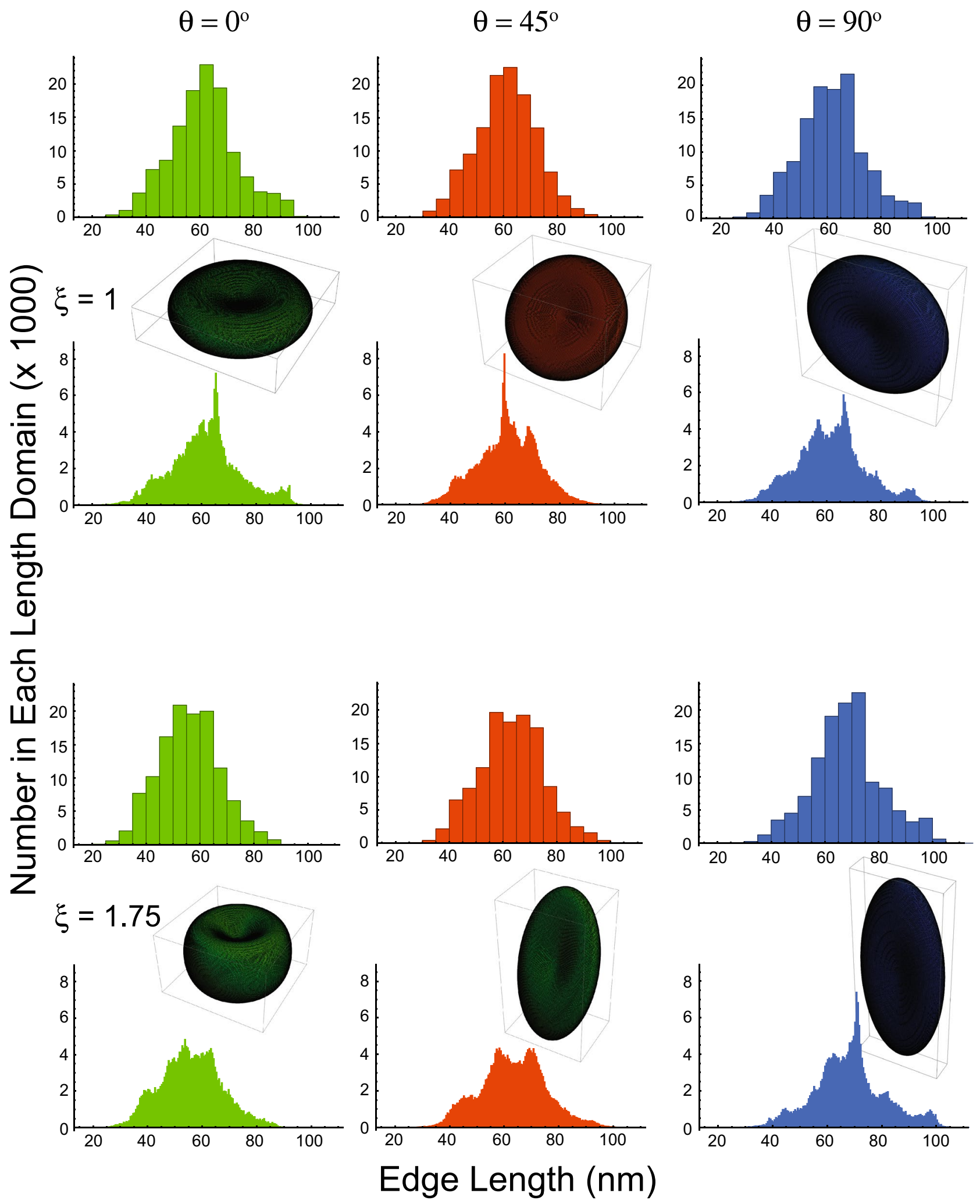

Figure 8. Histograms of the lengths of the 121,000 edges in the triangularization of an RBC at each of three rotations about the $x$-axis with no stretching $(\xi=1)$, and $75 \%$ stretching $(\xi=1.75)$. The colour coding was used to distinguish the three groups of data according to the rotations: green $0^{\circ}$, red $45^{\circ}$, and blue $90^{\circ}$. The bin numbers were 20 and 200 for the upper and lower pair of histograms at each value of stretching, $\xi$, respectively. The insets show the RBC shapes from which the histograms were made; they are the same as given in Figs. 4, S4 and S5. 
Figure 9. Partitioning of mesh-triangles of an RBC into those that underwent an increase in area and those that were diminished when the cell was strained in the $\mathrm{z}$-direction. The RBCs had an angle of orientation to the strain field of $\theta=0^{\circ}$ and $\theta=45^{\circ}$, respectively, prior to being stretched by the factor $\xi=1.75$. In order to speed up computation the triangularization was performed with 13,188 edges and not the real $\sim 121,000$. The Mathematica Notebook was written to achieve direct correspondence between a triangle in the relaxed RBC and its counterpart in the stretched state. (a) Shows the mesh-triangles that were increased in area; (b) those that were diminished in area; and (c) is a union of (a) and (b), which shows complete closure of the mesh by the two classes of triangles. For the $\theta=45^{\circ}$ orientation, (d) shows the mesh-triangles that were increased in area. Note how these occupy two disconnected manifolds, while (e) is the single perforated surface that contains those mesh-triangles that were diminished in area; and (f) is the union of the two classes of triangles. Notebook 7 contains the computations not only for this figure but for mapping the differences in the four curvatures between the relaxed and stretched RBCs.

triangle of $200 \mathrm{~nm}$ on each side has an area of $0.0173 \mu \mathrm{m}^{2}$. With a closed single-sheet surface like the RBC, each edge in the tessellation is shared with two triangles, and since there are three edges per triangle, the number of triangles is $2 / 3$ the number of edges. This implies that there are 80,667 triangles, which, when multiplied by $0.0173 \mu \mathrm{m}^{2}$, gives a total area of $1397 \mu \mathrm{m}^{2}$, a number 10.9 times greater than for the real/actual RBC.

On the other hand, if we assume there are 80,667 cytoskeletal triangles, this implies a triangle area of $128 / 80,667=0.0016 \mu \mathrm{m}^{2}$, which translates (using the triangle area formula above) to a side length of $60.8 \mathrm{~nm}$. In other words, the internodal distance of the RBC cytoskeletal network should be $\sim 60 \mathrm{~nm}$. This number compares favourably with what we obtained as the mean distance of the edges in the tessellation analysed in Fig. 2: viz., $75 \%$ of the edges span $60-70 \mathrm{~nm}$.

While the observation of a less-than-fully-extended triangular spectrin mesh could have been deduced without the complicated triangularization used here, the analysis nevertheless adds credibility to the ab initio triangularization process; while the discussion above confirms the consistency of the numerical values reported in the literature with a (fairly) regular triangular tessellation ${ }^{24}$.

Curvature. In its present form, the analysis of curvature and its graphical representation conveys a semiquantitative impression of how the distorted RBCs might transmit the locally altered shape of the membrane to Piezo1. However, the range-of-influence of membrane curvature on Piezol remains to be determined. Perhaps a finer mesh of triangularization is needed to explore this. The approaches adopted here should be extendable to such situations.

At the level of formal 3D differential geometry it was important to check for conformity of the total curvature with the Gauss-Bonnet Theorem ${ }^{17}$, which states that the integral of the Gaussian curvature over a closed surface will be $4 \pi$ for surfaces like the RBC, even with its dimples that have regions of negative curvature. Notebook 5 shows an example of this outcome in which the Gaussian curvature in each triangle was multiplied by the area of the respective triangle and then the sum taken over them all. This is tantamount to a finite difference approximation to the surface integral and the result was a gratifying verification of the overall curvature analysis.

Mesh-triangle area expansion and diminution. Figure $9 \mathrm{a}-\mathrm{c}$ are examples of the partitioning of meshtriangles into those that are expanded and those that are diminished when the RBC is exposed to the linear strain field at $0^{\circ}$. On the other hand, with the RBC at $45^{\circ}$ (Figs. 9d-f) the pattern of the mesh-triangles that are expanded or diminished is quite different. In the former case, the expanded triangles are arrayed in a single ring, while in the latter case, there are two separate regions each with two holes in them. For the diminished triangles in the $\mathrm{RBC}$ at $0^{\circ}$ (Fig. 9b), there are two separate continuous zones; and for the $\mathrm{RBC}$ at $45^{\circ}$, there is one centrally perforated sheet. If there were an uneven distribution of Piezol throughout the RBC membrane, say in the dimples as has been suggested by Svetina et al. ${ }^{35}$, then the action of Piezo1 would depend on the orientation of the RBCs to the strain field. Hence, it is not obvious whether the net effect of distortion of a randomly orientated population of such RBCs would actually lead to net activation of Piezo1.

Numerous studies have shown that Piezol responds to an increase in membrane tension ${ }^{7,36,37}$. Ion channel reconstitution into lipid bilayers has revealed that many eukaryotic channels respond to membrane tension ${ }^{38}$. The idea that ion channels can respond to membrane tension originates from the pioneering studies of bacterial mechanosensitive channels $\mathrm{s}^{39,40}$; and this has been underscored recently by work using a membrane-tension device with KcsA, that had not previously been considered to be mechanosensitive ${ }^{41}$. Hence, it is conceivable that membrane transporters other than Piezol in RBCs could be affected by shape changes of the type modelled here.

It is proposed that expansion of a mesh-triangle corresponds to an increase in local tension, whereas a decrease in area corresponds to relaxation. Therefore, from the numerous studies mentioned above, we conclude that even if Piezol were uniformly distributed around the RBC membrane, then members of the channel population would not be uniformly activated (at a given orientation to the direction of the strain field). This is further complicated by the non-uniform local levels of cytoskeletal contractility in RBCs, which will also influence local membrane tension ${ }^{42}$. Thus, the net extent of activation of the Piezol channels would depend on the orientation of each RBC to the strain field, and to local levels of RBC contractility. This would make the idea of segregation of Piezol to the pits of the RBC dimples less plausible ${ }^{35}$. Of course, studies involving light microscopy used with fluorescent antibodies directed specifically at Piezol on RBCs could settle this question. 

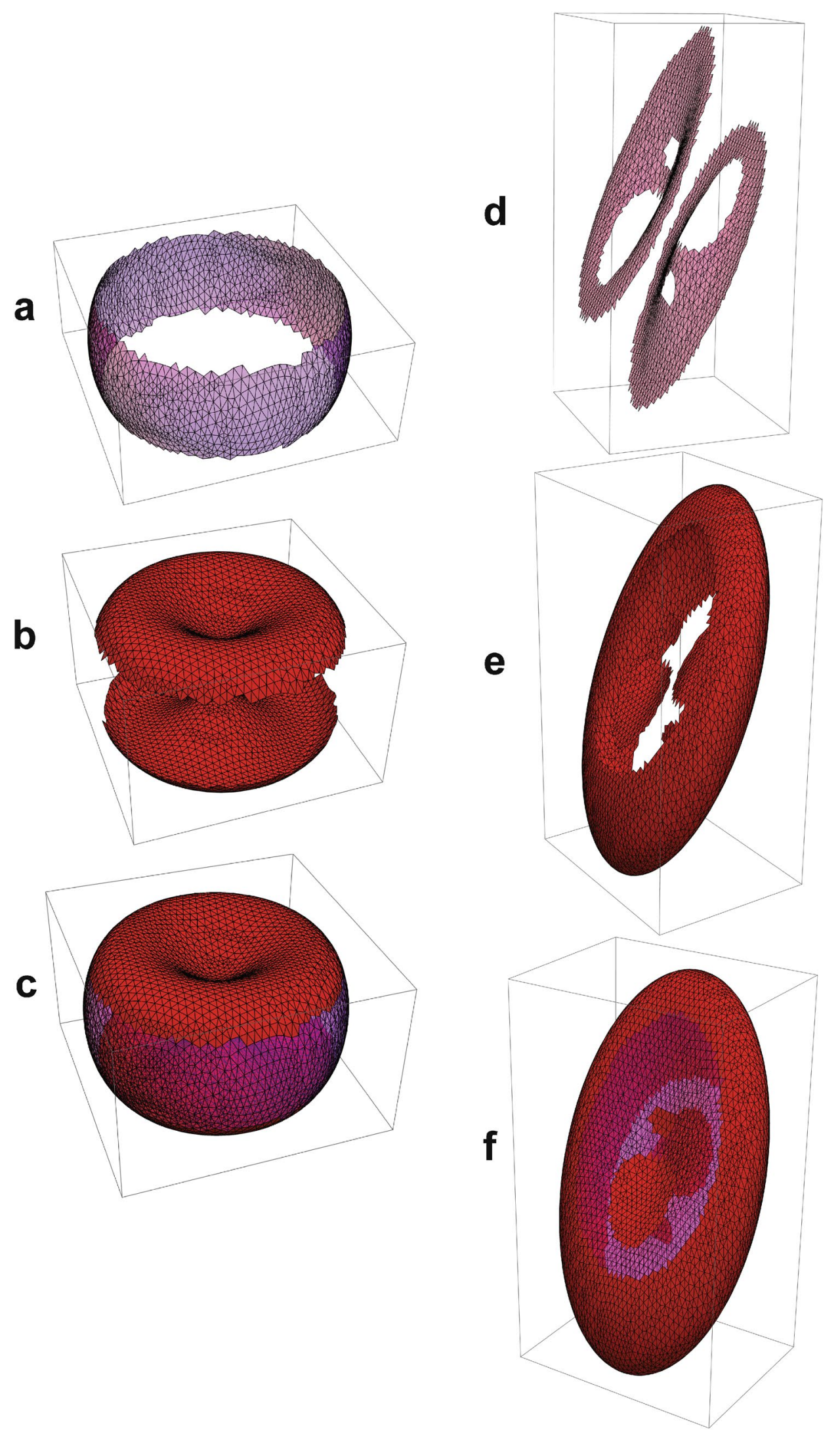
Cytoskeletal rearrangement. Since our pictures of RBCs, in various states of distortion are static, we can deduce nothing about the kinetics of cytoskeletal rearrangement of a kind suggested by Gov et al. ${ }^{43}$. However, the images of positive and negative changes in area of mesh-triangles (Fig. 9) suggest that rearrangements of the struts of the geodetic dome would occur such that strain would be re-distributed more uniformly. Whether this rearrangement occurs on the timescale of the blood circulation, or even during the $\sim 0.3 \mathrm{~s}$ taken to pass through a capillary in the human body ${ }^{44}$, requires more experimentation.

\section{Conclusions}

There are many aspects of RBC shape, volume and flexibility that are ripe for explanation ${ }^{45,46}$, and inevitably these studies will tap into recent findings on Piezo1, and new analytical methods including computational fluid dynamics.

At a deeper conceptual level, the work described here is an exploration of the geometrical constraints on 'biological form' (e.g., ${ }^{28,47}$ ); in this case, the particular shapes are those taken up by an RBC in a strain field. The linear strain field used here is the simplest of all, being in a single direction: but it is consistent with that imposed by a stretched gel, as used in our NMR experiments ${ }^{15}$. Much more complicated deformations occur in flowing systems, in which some domains of the RBC membrane are stressed into more positive curvature, while others simultaneously undergo more negative curvature ${ }^{48-51}$. The ability of the RBC to accommodate these contortions decreases with the age of the cell and it is posited as a major factor in what determines RBC survival, for $\sim 120$ days in the circulation ${ }^{33}$. Transient, distorted shapes exist in RBCs when they are in regions of high velocity that impose non-laminar flow around prosthetic or even healthy heart valves. Flow changes occur during valve development in cardio-genesis in particular, and flow is modified around calcified or diseased valves, not just prosthetic ones ${ }^{50,51}$; so, there is considerable merit in having a computationally accessible means of modelling RBC shape changes by using the methods presented here.

The RBC shapes in various in vivo situations have begun to yield to computation. For example, it is known that the stresses on an RBC can be so extreme around prostheses as to lead to cell rupture. To date, simulating such outcomes really only yield to advanced supercomputing e.g., ${ }^{52}$.

The other critical aspect of simulations for surface deformation is the distance scale of the deformations that are required to activate mechanosensitive ion channels like Piezo $1^{11,14}$. The changes in curvature invoked at the tip of a patch-clamp pipette are quite extreme, across a diameter of $\sim 1 \mu \mathrm{m}$ or an area of $0.79 \mu \mathrm{m}^{2}$, implying that 80,667 (number of triangles) $\times 0.79\left(\mu \mathrm{m}^{2}\right.$, area of patch) $/ 128\left(\mu \mathrm{m}^{2}\right.$, total area of $\left.\mathrm{RBC}\right) \cong 500$ cytoskeletal triangles are spanned. The resolution of the present simulations and the curvature near the rim of the RBC are in the same range. Therefore, if increased membrane bending activates Piezo1, then it will be those molecules at the rim of the cells (as in Fig. 6, right) that would be activated. On the other hand, if a decrease in curvature (increased area of flatness) is what activates them, then it will be also found in the stretched cell. Even more interesting is the fact that the two Principal Curvatures $\mathrm{k}_{1}$ and $\mathrm{k}_{2}$ both increase on stretching the cell (Fig. 6 right).

However (as alluded to in the "Introduction"), in patch clamping experiments the visible curvature appears not to be what activates the channel. The inflation of the membrane dome is driven by the confinement by the micro-pipette that is on the micron scale. It is the tension (and presumably flattening at the nm scale below the resolution of a confocal microscope) that appears to drive the channels to open ${ }^{14,37,53-55}$. Whereas in stretched/ compressed gel experiments, the morphological forms taken up by the RBCs will be like those shown in this article. However, more curvature of the membrane on the length scales seen in membrane flickering ${ }^{56,57}$ would be superimposed on these shapes.

Future directions for this computational work will involve larger scale simulations of population-averaged curvatures in cells under strain, and in strain fields that are not simply unidirectional and linear. Then, correlations might be able to be made with experimental measurements like those already reported on stretched/compressed gels ${ }^{1,2}$, including estimates of membrane tension during electrophysiological measurements performed on whole RBCs.

Received: 27 April 2021; Accepted: 7 June 2021

Published online: 01 July 2021

\section{References}

1. Kuchel, P. W. et al. Enhanced $\mathrm{Ca}^{2+}$ influx in mechanically distorted erythrocytes measured with ${ }^{19} \mathrm{~F}$ nuclear magnetic resonance spectroscopy. Sci. Rep. 11(1), 83044 (2021).

2. Kuchel, P. W. \& Shishmarev, D. Accelerating metabolism and transmembrane cation flux by distorting red blood cells. Sci. Adv. 3(10), 1016 (2017)

3. Coste, B. et al. Piezo1 and Piezo2 are essential components of distinct mechanically-activated cation channels. Science 330(6000), 55-60 (2010).

4. Ranade, S. S. et al. Piezo1, a mechanically activated ion channel, is required for vascular development in mice. Proc. Natl. Acad. Sci. USA. 111(28), 10347-10352 (2014).

5. Archer, N. M. et al. Hereditary xerocytosis revisited. Am. J. Hematol. 89(12), 1142-1146 (2014).

6. Cahalan, S. M. et al. Piezo1 links mechanical forces to red blood cell volume. eLife 4, 7370 (2015).

7. Syeda, R. et al. Piezol channels are inherently mechanosensitive. Cell Rep. 17(7), 1739-1746 (2016).

8. Evans, E. L. et al. RBCs prevent rapid PIEZO1 inactivation and expose slow deactivation as a mechanism of dehydrated hereditary stomatocytosis. Blood 136(1), 140-144 (2020).

9. Rotordam, M. G. et al. A novel gain-of-function mutation of Piezo1 is functionally affirmed in red blood cells by high-throughput patch clamp. Haematologica 104(5), E179-E183 (2019).

10. Gottlieb, P. A., Bae, C. \& Sachs, F. Gating the mechanical channel Piezo1: A comparison between whole-cell and patch recording. Channels 6(4), 282-289 (2012).

11. Guo, Y. R. \& MacKinnon, R. Structure-based membrane dome mechanism for Piezo mechanosensitivity. eLife 6, e33660 (2017). 
12. Wang, Y. \& Xiao, B. The mechanosensitive Piezol channel: structural features and molecular bases underlying its ion permeation and mechanotransduction. J. Physiol. 596(6), 969-978 (2017).

13. Saotome, K. et al. Structure of the mechanically activated ion channel Piezol. Nature 554(7693), 481-486 (2018).

14. Lin, Y. C. et al. Force-induced conformational changes in PIEZO1. Nature 573(7773), 230-234 (2019).

15. Shishmarev, D., Momot, K. I. \& Kuchel, P. W. Anisotropic diffusion in stretched hydrogels containing erythrocytes: Evidence of cell-shape distortion recorded by PGSE NMR spectroscopy. Magn. Reson. Chem. 55(5), 438-446 (2017).

16. Kuchel, P. W. \& Fackerell, E. D. Parametric-equation representation of biconcave erythrocytes. Bull. Math. Biol. 61(2), 209-220 (1999).

17. Abbena, A., Salamon, S. \& Gray, A. Modern Differential Geometry of Curves and Surfaces with Mathematica (Chapman and Hall/ CRC, 2006).

18. Goldman, R. Curvature formulas for implicit curves and surfaces. Comput. Aided Geom. Des. 22, 632-658 (2005).

19. Mathematica. Wolfram Research, Inc. (www.wolfram.com). Mathematica Online (2020).

20. Wolfram, S. The Mathematica Book 5th edn. (Wolfram Media Inc., 2003).

21. Svetina, S. \& Zeks, B. Membrane bending energy and shape determination of phospholipid vesicles and red blood cells. Eur. Biophys. J. 17, 101-111 (1989).

22. Grimes, A. J. Human Red Cell Metabolism (Blackwell Scientific, 1980).

23. Svetina, S. Relations among variations in human red-cell volume, density, membrane area, hemoglobin content and cation content. J. Theor. Biol. 95(1), 123-134 (1982).

24. Lux, S. E. Anatomy of the red cell membrane skeleton: unanswered questions. Blood 127(2), 187-199 (2016).

25. Sakurai, J. J. \& Napolitano, J. Modern Quantum Mechanics 2nd edn. (Addison-Wesley, 2011).

26. Lipschutz, S. Schaum's Outline of Theory and Problems of Linear Algebra (McGraw-Hill Book Company, 1968).

27. Spiegel, M. R. Schaum's Outline of Theory and Problems of Vector Analysis (McGraw-Hill Publishing Co., 1959).

28. Coxeter, H. S. M. Introduction to Geometry (Wiley, 1969).

29. Pan, L. et al. Super-resolution microscopy reveals the native ultrastructure of the erythrocyte cytoskeleton. Cell Rep. 22, 1151-1158 (2018).

30. Rogers, S. \& Lew, V. L. Up-down biphasic volume response of human red blood cells to PIEZO1 activation during capillary transits. PLoS Comput. Biol. 17, 1-18 (2021).

31. Rogers, S. \& Lew, V. L. PIEZO1 and the mechanism of the long circulatory longevity of human red blood cells. PLoS Comput. Biol. $17,1-22(2021)$.

32. Ponder, E. Hemolysis and Related Phenomena (J. \& A. Churchill Ltd., 1948).

33. Lew, V. L. \& Tiffert, T. On the mechanism of human red blood cell longevity: Roles of calcium, the sodium pump, PIEZO1, and Gárdos channels. Front. Physiol. 8, 0977 (2017).

34. Bae, C. et al. Xerocytosis is caused by mutations that alter the kinetics of the mechanosensitive channel PIEZO1. Proc. Natl. Acad. Sci. USA 110(12), E1162-E1168 (2013).

35. Svetina, S., Kebe, T. S. \& Bozic, B. A model of piezo1-based regulation of red blood cell volume. Biophys. J. 116(1), 151-164 (2019).

36. Lewis, A. H. \& Grandl, J. Mechanical sensitivity of Piezo1 ion channels can be tuned by cellular membrane tension. eLife 4, e12088 (2015).

37. Cox, C. D. et al. Removal of the mechanoprotective influence of the cytoskeleton reveals PIEZO1 is gated by bilayer tension. Nat. Commun. 7, 10366 (2016).

38. Cox, C. D., Bavi, N. \& Martinac, B. Biophysical principles of ion-channel-mediated mechanosensory transduction. Cell Rep. 29(1), $1-12(2019)$.

39. Martinac, B., Adler, J. \& Kung, C. Mechanosensitive ion channels of E. coli activated by amphipaths. Nature 348(6298), 261-263 (1990).

40. Moe, P. \& Blount, P. Assessment of potential stimuli for mechano-dependent gating of MscL: Effects of pressure, tension, and lipid headgroups. Biochemistry 44(36), 12239-12244 (2005).

41. Iwamoto, M. \& Oiki, S. Hysteresis of a tension-sensitive $\mathrm{K}^{+}$channel revealed by time-lapse tension. J. Am. Chem. Soc. 1, 467-474 (2021).

42. Alimohamadi, H. et al. Non-uniform distribution of myosin-mediated forces governs red blood cell membrane curvature through tension modulation. PLoS Comput. Biol. 16(5), e1007890 (2020).

43. Gov, N. S. \& Safran, S. A. Red blood cell membrane fluctuations and shape controlled by ATP-induced cytoskeletal defects. Biophys. J. 88, 1859-1874 (2005).

44. Gregersen, M. I. et al. Flow characteristics of human erythrocytes through polycarbonate sieves. Science 157, 825-827 (1967)

45. Hoffman, J. F. Reflections of the cracked timber of red blood cell physiology. Blood Cells Mol. Dis. 79, 102354 (2019).

46. Hoffman, J. F. Red blood cells, compasses and snap shots. Blood Cells Mol. Dis. 71, 67-70 (2018).

47. Thompson, D. W. On Growth and Form (Cambridge University Press, 1917).

48. Sui, Y. et al. Dynamic motion of red blood cells in simple shear flow. Phys. Fluids 20(11), 112160 (2008).

49. Chung, B. et al. Computational fluid dynamics of aggregating red blood cells in postcapillary venules. Comput. Methods Biomech. Biomed. Eng. 12(4), 385-397 (2009).

50. Yu, H. et al. A review of hemolysis prediction models for computational fluid dynamics. Artif. Organs 41(7), 603-621 (2017).

51. James, M. E., Papavassiliou, D. V. \& O’Rear, E. A. Use of computational fluid dynamics to analyze blood flow, hemolysis and sublethal damage to red blood cells in a bileaflet artificial heart valve. Fluids 4(1), 19 (2019).

52. Alexiadis, A., A new framework for modelling the dynamics and the breakage of capsules, vesicles and cells in fluid flow, in IUTAM Symposium on Dynamics of Capsules, Vesicles and Cells in Flow (D. BarthesBiesel, M.G. Blyth, \& A.V. Salsac, Eds). p. $80-88$ (2015).

53. Bavi, O. et al. Influence of global and local membrane curvature on mechanosensitive ion channels: A finite element approach. Membranes 6(1), 14 (2016).

54. Cox, C. D., Bavi, N. \& Martinac, B. Origin of the force: The force-from-lipids principle applied to piezo channels. In Piezo Channels (ed. Gottlieb, P. A.) 59-96 (2017).

55. Cox, C. D. \& Martinac, B. Bending Piezo1: The effect of amphipaths on the gating of a mechanosensitive channel. Biophys. J. 110(3), 349A-349A (2016).

56. Puckeridge, M. et al. Membrane flickering of the human erythrocyte: physical and chemical effectors. Eur. Biophys. J. Biophys. Lett. 43(4-5), 169-177 (2014).

57. Puckeridge, M. \& Kuchel, P. W. Membrane flickering of the human erythrocyte: Constrained random walk used with Bayesian analysis. Eur. Biophys. J. Biophys. Lett. 43(4-5), 157-167 (2014).

\section{Acknowledgements}

The work was funded by an Australian Research Council Discovery Project Grant DP1901005100 awarded to P.W.K., D.S., C.D.C., and P.G. D.S. also acknowledges Investigator Grant GNT1173015 from the Australian National Health and Medical Research Council. Drs Soumya Mohapatra and Duncan Pettengill, consultants at Wolfram Technology Group, are thanked for advice on 3D-surface triangularization and metrification using 
Mathematica. Professor Arthur Conigrave, University of Sydney, is thanked for valuable discussions on the RBC cytoskeleton. Drs Amin Rahmat and Associate Professor Alessio Alexiadis, Engineering, University of Birmingham, UK, are thanked for collaborative work on numerically simulated (computationally intensive) shape changes in RBCs in shear fields.

\section{Author contributions}

All authors (P.W.K., C.D.C., D.D., D.S., and P.G.) provided input into the plan of this Sub-Project (RBC shape simulation) of the ARC Discovery Project, DP190100510. This was jointly awarded to P.W.K., C.D.C., D.S., and P.G. D.D. engaged in valuable discussions on 3D differential geometry including especially Dr Ron Goldman's valuable article ${ }^{18}$. P.W.K. carried out the mathematical and computational analyses and drafted the manuscript, while all authors (P.W.K., C.D.C., D.D., D.S., and P.G.) contributed to the final version.

\section{Competing interests}

The authors declare no competing interests.

\section{Additional information}

Supplementary Information The online version contains supplementary material available at https://doi.org/ 10.1038/s41598-021-92699-7.

Correspondence and requests for materials should be addressed to P.W.K.

Reprints and permissions information is available at www.nature.com/reprints.

Publisher's note Springer Nature remains neutral with regard to jurisdictional claims in published maps and institutional affiliations.

(c) (i) Open Access This article is licensed under a Creative Commons Attribution 4.0 International License, which permits use, sharing, adaptation, distribution and reproduction in any medium or format, as long as you give appropriate credit to the original author(s) and the source, provide a link to the Creative Commons licence, and indicate if changes were made. The images or other third party material in this article are included in the article's Creative Commons licence, unless indicated otherwise in a credit line to the material. If material is not included in the article's Creative Commons licence and your intended use is not permitted by statutory regulation or exceeds the permitted use, you will need to obtain permission directly from the copyright holder. To view a copy of this licence, visit http://creativecommons.org/licenses/by/4.0/.

(C) The Author(s) 2021 